\title{
Incidence and Prognostic Value of the Systemic Inflammatory Response Syndrome and Organ Dysfunctions in Ward Patients
}

\author{
Matthew M. Churpek ${ }^{1}$, Frank J. Zadravecz ${ }^{1}$, Christopher Winslow ${ }^{2}$, Michael D. Howell ${ }^{1}$, and Dana P. Edelson ${ }^{1}$ \\ ${ }^{1}$ Department of Medicine, University of Chicago, Chicago, Illinois; and ${ }^{2}$ Department of Medicine, NorthShore University HealthSystem, \\ Evanston, Illinois
}

\begin{abstract}
Rationale: Tools that screen inpatients for sepsis use the systemic inflammatory response syndrome (SIRS) criteria and organ dysfunctions, but most studies of these criteria were performed in intensive care unit or emergency room populations.
\end{abstract}

Objectives: To determine the incidence and prognostic value of SIRS and organ dysfunctions in a multicenter dataset of hospitalized ward patients.

Methods: Hospitalized ward patients at five hospitals from November 2008 to January 2013 were included. SIRS and organ system dysfunctions were defined using 2001 International Consensus criteria. Patient characteristics and in-hospital mortality were compared among patients meeting two or more SIRS criteria and by the presence or absence of organ system dysfunction.
Measurements and Main Results: A total of 269,951 patients were included in the study, after excluding 48 patients with missing discharge status. Forty-seven percent $(n=125,841)$ of the included patients met two or more SIRS criteria at least once during their ward stay. On ward admission, 39,105 (14.5\%) patients met two or more SIRS criteria, and patients presenting with SIRS had higher inhospital mortality than those without SIRS (4.3\% vs. $1.2 \%$; $P<0.001)$. Fourteen percent of patients $(n=36,767)$ had at least one organ dysfunction at ward admission, and those presenting with organ dysfunction had increased mortality compared with those without organ dysfunction (5.3\% vs. $1.1 \% ; P<0.001)$.

Conclusions: Almost half of patients hospitalized on the wards developed SIRS at least once during their ward stay. Our findings suggest that screening ward patients using SIRS criteria for identifying those with sepsis would be impractical.

Keywords: systemic inflammatory response syndrome; sepsis; multiple organ failure; organ dysfunction scores
The systemic inflammatory response syndrome (SIRS) was introduced by Bone and colleagues (1) during a 1991 consensus conference with the goals of improving the early detection of patients with sepsis, facilitating standardization of research protocols, and providing useful prognostic information. Since that time, the SIRS criteria have been widely adopted in both research and clinical practice $(2,3)$. In addition, definitions of organ dysfunction have been developed that both define the syndrome of severe sepsis and provide prognostic value $(1,4-6)$. However, the utility of SIRS has been questioned, with most arguments focusing on the criteria's low specificity (7). For example, many other noninfectious conditions can result in the development of SIRS, such as pancreatitis, myocardial infarction, and pulmonary embolism (1). In addition, even exercise can result in meeting the heart rate and respiratory rate portions of the SIRS criteria. However, the SIRS criteria are still commonly used in sepsis bundles and as entry criteria for clinical trials $(2,3,8,9)$.

(Received in original form February 9, 2015; accepted in final form July 9, 2015)

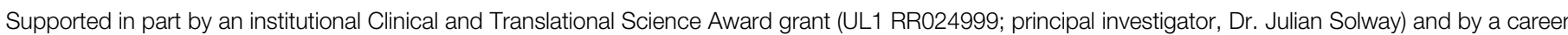
development award from the NHLBI (K08 HL121080) (M.M.C.)

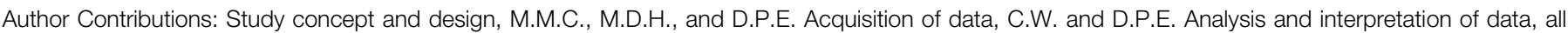

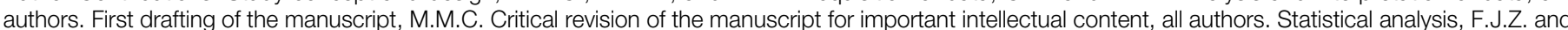

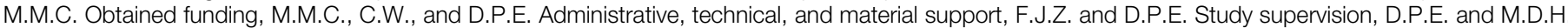

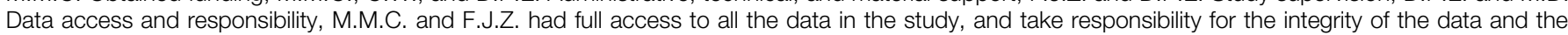
accuracy of the data analysis.

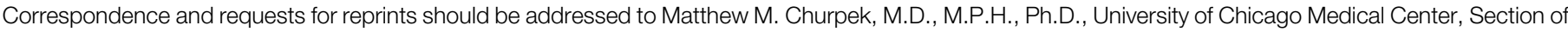
Pulmonary and Critical Care Medicine, 5841 South Maryland Avenue, MC 6076, Chicago, IL 60637. E-mail: matthew.churpek@uchospitals.edu

This article has an online supplement, which is accessible from this issue's table of contents at www.atsjournals.org

Am J Respir Crit Care Med Vol 192, Iss 8, pp 958-964, Oct 15, 2015

Copyright (C) 2015 by the American Thoracic Society

Originally Published in Press as DOI: 10.1164/rccm.201502-02750C on July 9, 2015

Internet address: www.atsjournals.org 


\section{At a Glance Commentary}

\section{Scientific Knowledge on the}

Subject: The systemic inflammatory response syndrome (SIRS) and organ dysfunction criteria are often used as part of sepsis screening tools. However, the incidence and prognostic value of these criteria have been primarily studied in intensive care unit and emergency department patients.

\section{What This Study Adds to the}

Field: We found that almost half of patients hospitalized on the wards developed SIRS at least once during their ward stay. In-hospital mortality was higher for patients who presented to the wards with SIRS or any organ dysfunction and increased with the number of criteria met. Our findings suggest that screening ward patients using SIRS criteria for identifying sepsis would be time-consuming and impractical.

To date, most studies on SIRS and sepsis-related organ dysfunction have been performed in the intensive care unit (ICU) and the emergency department $(4,10-13)$. However, further investigation into the incidence and prognosis associated with these criteria in ward patients is essential for several reasons. First, many patients who develop sepsis are initially treated on the wards (14), and it has been shown that patients admitted to the ICU from the wards have a higher mortality rate than those admitted to the ICU from the emergency department $(15,16)$. Second, many hospitals are now expanding their sepsis screening programs to the wards (17-19), and so information regarding the incidence and prognostic value of these criteria would inform these efforts. Finally, the evolution of different organ dysfunctions over time on the wards may impart important prognostic information for these patients. Therefore, we aimed to investigate the incidence and prognostic value of SIRS, organ dysfunctions, and their evolution over time in a multicenter dataset of hospitalized ward patients. Some of the results have been previously reported in the form of an abstract (20).

\section{Methods}

\section{Study Population}

All adult patients hospitalized at four NorthShore University HealthSystem hospitals (Evanston, Glenbrook, Highland Park, and Skokie) and the University of Chicago from November 2008 to January 2013 with documented ward vital signs were included in the study. All hospitals admit both medical and surgical patients, and additional details for the study hospitals are shown in Table E1 in the online supplement (21). Patients were excluded from the study if they were missing in-hospital mortality data. In addition, only observations occurring during a patient's initial ward stay were used in this study, and observations were censored at the time of discharge, death, or first ward to ICU transfer. The study protocol was approved, with a waiver of consent granted based on minimal harm and general impracticability, by the University of Chicago Institutional Review Board (IRB \#16995A) and NorthShore University HealthSystem (IRB \#EH11-258).

\section{Data Collection}

Patient demographics, location- and timestamped vital signs (blood pressure, heart rate, respiratory rate, oxygen saturation, temperature, and mental status [coded as alert, responds to voice, responds to pain, or unresponsive (AVPU)]), and laboratory results were obtained from the Clinical Research Data Warehouse at the University of Chicago and the Electronic Data Warehouse at NorthShore. Vital sign frequency in the data represented standard practice at each hospital, and was typically every 4 hours unless the care team ordered a different frequency. Laboratory data were collected at the discretion of the treating physician, which typically included laboratory testing every 24 hours. Vital sign data were entered manually into the electronic health record using a bedside computer, a computer on wheels, or a central desktop computer, and laboratory data were automatically uploaded by the hospital's laboratory results system. The time the vital signs and laboratory results were entered into the electronic medical record was used as the observation time for each value in the dataset. These data were then available for acquisition from each hospital's data warehouse. Nonphysiologic values (i.e., respiratory rate $>70$ or $<1$ breaths $/ \mathrm{min}$, heart rate $>300$ or $<1$ beats $/ \mathrm{min}$, temperature $>44^{\circ} \mathrm{C}$ or $<32^{\circ} \mathrm{C}$ ) were changed to missing for the purposes of this study.

\section{SIRS and Organ Dysfunction Definitions}

SIRS criteria were defined according to updates from the 2001 International Consensus conference (i.e., temperature $>38^{\circ} \mathrm{C}$ or $<36^{\circ} \mathrm{C}$; heart rate $>90$ beats/ $\mathrm{min}$; respiratory rate $>20$ breaths/min or $\mathrm{Pa}_{\mathrm{CO}_{2}}<32 \mathrm{~mm} \mathrm{Hg}$; white blood cell count $>12,000 / \mathrm{mm}^{3},<4,000 / \mathrm{mm}^{3}$, or $>10 \%$ immature neutrophils [bands]) $(1,22)$. The $\mathrm{Pa}_{\mathrm{CO}_{2}}$ and immature band criteria were not available and therefore were not used in the study. A patient was considered to have SIRS if any two of the defined criteria were met simultaneously. Organ dysfunction criteria were defined by system, and adapted from 2001 consensus definitions as follows: neurologic (an observation of "responds to voice," "responds to pain," or "unresponsive" on the AVPU scale), hematologic (a platelet count $<100 \mathrm{k} / \mathrm{mm}^{3}$ ), renal (a creatinine value $>2.0 \mathrm{mg} / \mathrm{dl}$ ), pulmonary (a respiratory rate $>24$ breaths/min, or an oxygen saturation reading $<90 \%$ ), metabolic (an anion gap value $>16$ ), cardiovascular (a systolic blood pressure reading $<90 \mathrm{~mm} \mathrm{Hg}$ ), and hepatic (a total bilirubin value $>4 \mathrm{mg} / \mathrm{dl}$ ) $(1,22)$. Of note, the tachypnea threshold for pulmonary dysfunction was increased to greater than 24, as per the Acute Physiology and Chronic Health Evaluation III reference range, so as to not overlap with the definition used for SIRS (23). For each observation time, any missing values for SIRS or organ dysfunction determination were pulled forward from the most recent previous value during the current admission. If no prior values were available, then a normal (i.e., median) value was imputed. This practice is similar to previous studies and reflects the fact that vital signs are collected more frequently than laboratory values $(21,23,24)$.

\section{Statistical Analysis}

Demographic characteristics were compared among patients who ever developed SIRS during their ward stay and those who never developed SIRS. In addition, the relationship between inhospital mortality and the individual and cumulative number of SIRS criteria were investigated at ward admission. A graph of the cumulative proportion of patients remaining on the wards who met SIRS 
criteria at least once during their ward stay before each time point was constructed using a best-fit multivariable fractional polynomial function (25). The associations between mortality and each individual organ system dysfunction, the number of simultaneous organ dysfunctions, and the change over time of each organ dysfunction over the first 24 hours since ward admission were compared. For the change in organ dysfunction over time comparisons, time of ward admission was defined as the time of the first vital sign observed in a hospital ward location. The earliest set of observations measured at least 24 hours since admission was used for the change over time analyses. In addition, a sensitivity analysis was performed where only vital signs measured simultaneously were used for calculating whether a patient had SIRS and no previous or missing values were imputed. In the sensitivity analysis, white blood cell count was only allowed to be carried forward a maximum of 24 hours. A second sensitivity analysis where white blood cell count was only allowed to be carried forward a maximum of 1 hour was also performed.

Patient characteristics were compared using $t$ tests, chi-square tests, and Wilcoxon rank sum tests where appropriate. Chi-square tests were used to compare the proportion of patients who failed to survive to hospital discharge for each SIRS and organ dysfunction analysis. All analyses were performed using Stata version 12.1 (Stata Corporation, College Station, TX). A twotailed $P$ less than 0.05 denoted statistical significance for all comparisons.

\section{Results}

\section{Study Population and SIRS during Ward Stay}

A total of 269,951 patients were included in the study, after removing patients with a missing discharge status $(\mathrm{n}=48)$. In addition, 726 respiratory rate, 153 heart rate, and 370 temperature values were nonphysiologic and thus changed to missing $(<0.01 \%$ of values). The median for time between sequential values in the same patient was 3 hours (interquartile range [IQR], 1-5) for heart rate, 4 hours (IQR, 1-5) for respiratory rate, 4 hours (IQR, 3-6) for temperature, and 24 hours (IQR, 18-25) for white blood cell count. Forty-seven percent $(n=125,841)$ of the included patients met two or more SIRS criteria simultaneously at least once during their ward admission. Thirty-one percent of these patients $(n=39,105)$ presented with SIRS first at ward admission, whereas the remaining 69\% $(\mathrm{n}=86,736)$ developed SIRS after their first ward observation. The cumulative proportion of patients who met SIRS criteria at least once during their ward stay increased from $15 \%$ on ward admission to more than $70 \%$ for those patients remaining on the wards for 7 days (Figure 1). Of the 144,110 patients who never developed SIRS during their initial ward admission, $98 \%$ $(n=141,471)$ were censored because of being discharged from the hospital and the remaining $2 \%(n=2,639)$ were censored because of transfer to the ICU or death on the wards.

The median length of stay on the wards for patients discharged before developing SIRS was 53 hours (IQR, 33-84 h). When compared with those never having SIRS, patients who developed SIRS were more likely to be female ( $62 \%$ vs. $58 \%$ ), more likely to be black ( $22 \%$ vs. $15 \%$ ), and more likely to have multiple admissions during the study period compared with those without SIRS (median, 2 [IQR 1-4] vs. 2 [IQR 1-3]; $P<0.001$ for all comparisons) (Table 1). Additionally, patients who developed SIRS had a longer median hospital length of stay ( $4 \mathrm{~d}$ vs. $3 \mathrm{~d}$ ) and higher rate of in-hospital mortality (3\% vs. $0.5 \%)$ than those never developing SIRS ( $P<0.001$ for all comparisons).

\section{SIRS on Ward Admission}

On ward admission, $50 \%$ of the patients did not meet any SIRS criterion, 35\% met one criterion, $12 \%$ met two criteria, $2 \%$ met three criteria, and $0.2 \%$ met all four criteria. Patients who met at least two SIRS criteria at the time of admission had higher in-hospital mortality than those without SIRS (4.3\% vs. $1.2 \% ; P<0.001$ ). Also, in-hospital mortality increased with each additional SIRS criterion met, with $1.8 \%$ mortality if one of the criteria was met on admission up to $9.0 \%$ if all four criteria were met $(P<0.001$ for trend) (Figure 2$)$. Meeting the respiratory rate criteria of SIRS definition on admission had the highest associated in-hospital mortality $(6.2 \%)$ when compared with the heart rate (3.0\%), temperature $(2.5 \%)$, and white blood cell $(2.2 \%)$ criteria $(P<0.001$ for all comparisons).

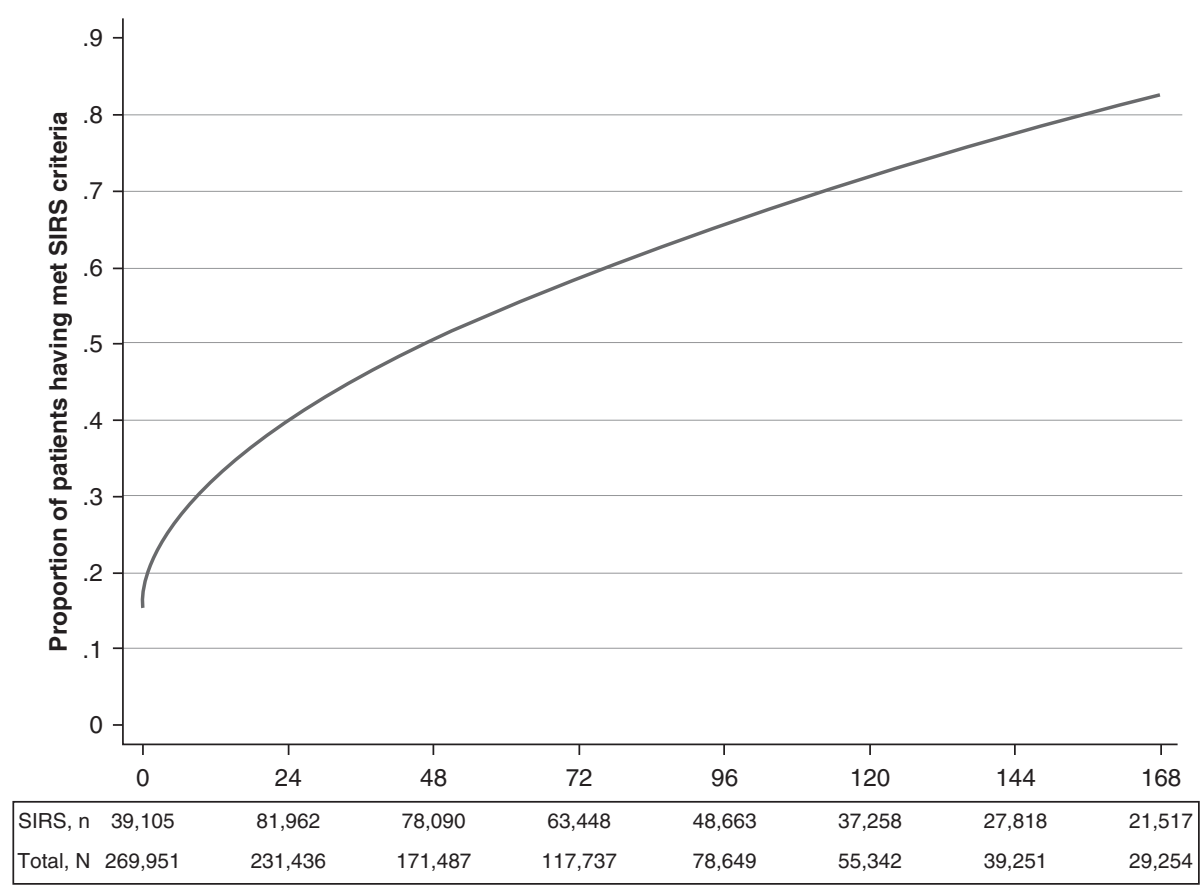

Time on the wards (hours)

Figure 1. Cumulative proportion of patients remaining on the wards who met systemic inflammatory response syndrome (SIRS) criteria at least once during their ward stay before each time point. 
Table 1. Comparison of Patient Characteristics between Those Who Developed SIRS during Their Ward Admission and Those Who Never Developed SIRS

\begin{tabular}{lcrl}
\hline & $\begin{array}{c}\text { SIRS during } \\
\text { Admission } \\
(\boldsymbol{n}=\mathbf{1 2 5 , 8 4 1 )}\end{array}$ & $\begin{array}{c}\text { No SIRS during } \\
\text { Admission } \\
(\boldsymbol{n}=\mathbf{1 4 4 , 1 1 0 )}\end{array}$ & $\boldsymbol{P}$ Value \\
Age, mean (SD), yr & $61(20)$ & $60(20)$ & $<0.001$ \\
Female sex, n (\%) & $89,594(62)$ & $72,686(58)$ & $<0.001$ \\
Race & $28,254(22)$ & $21,414(15)$ & $<0.001$ \\
$\quad$ Black, n (\%) & $63,408(50)$ & $77,019(53)$ & \\
White, n (\%) & $34,179(27)$ & $45,677(32)$ & \\
$\quad$ Other/unknown, n (\%) & $4(3-7)$ & $3(2-4)$ & $<0.001$ \\
Hospital length of stay, median (IQR), d & $3,873(3)$ & $665(0.5)$ & $<0.001$ \\
Died during hospital stay, n (\%) & &
\end{tabular}

Definition of abbreviations: IQR = interquartile range; $\mathrm{SIRS}=$ systemic inflammatory response syndrome.

\section{Sensitivity Analyses}

In the sensitivity analysis that used only simultaneously collected vital signs, a total of $3,585,716$ observation sets in 238,958 unique patient admission were included (35\% and $89 \%$ of the original cohort, respectively). Findings were similar to the main results of the study, with $47 \%$ of patients meeting SIRS criteria during their ward stay and $38 \%$ of these patients meeting SIRS criteria at their first ward observation. Associations with in-hospital mortality were also similar, with 3.3\% in-hospital mortality for those meeting two out of four SIRS criteria on admission versus $0.8 \%$ for those not meeting SIRS criteria. In-hospital mortality increased with each increasing number of SIRS criteria met on admission in the sensitivity analysis as well, from $0.5 \%$ mortality if none of the criteria were met to $9.2 \%$ if all four were met. Results were similar after further restricting the analysis and white blood cell count results were available within 1 hour, with $56 \%$ of patients meeting at least two of four SIRS criteria during their ward stay and a $4 \%$ in-hospital mortality rate for those meeting SIRS criteria on admission compared with $0.8 \%$ for those not meeting SIRS criteria.

\section{Organ Dysfunction on Admission and 24 Hours after Admission}

Fourteen percent of patients $(n=36,767)$ had at least one organ dysfunction at ward admission, and the presence of any organ dysfunction at admission was associated with increased in-hospital mortality (5.3\% to times where vital signs were simultaneous

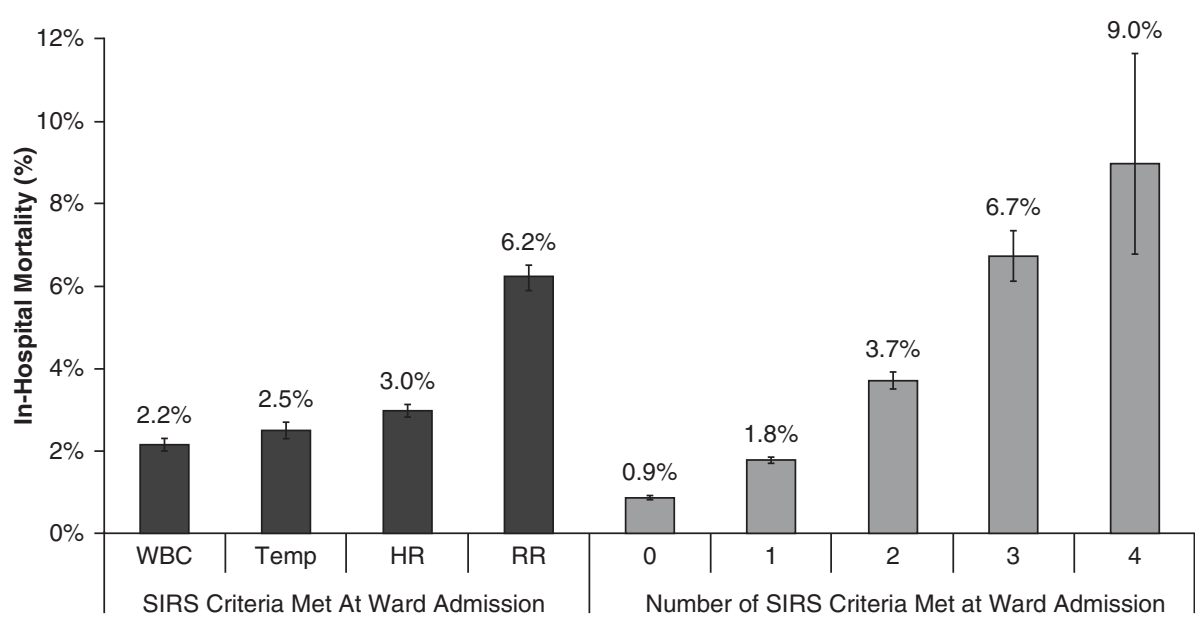

Figure 2. The association between in-hospital mortality and systemic inflammatory response syndrome (SIRS) criteria met at ward admission. $\mathrm{HR}=$ heart rate; $\mathrm{RR}=$ respiratory rate; Temp = temperature; $\mathrm{WBC}=$ white blood cell count. vs. $1.1 \% ; P<0.001)$. Of the individual organ systems, pulmonary organ dysfunction was associated with the highest risk of death before hospital discharge (13.2\%). Although the presence of neurologic dysfunction at ward admission had the third-highest risk of in-hospital mortality $(6.3 \%)$ after pulmonary dysfunction and cardiovascular dysfunction (12.3\%), the presence of neurologic dysfunction at 24 hours had a higher association with in-hospital mortality than any other organ system $(19.9 \% ; P<0.001$ for all comparisons). A higher percentage of patients met organ dysfunction criteria at 24 hours after ward admission (15.3\%) than at admission $(P<0.001)$. As shown in Figure 3, in-hospital mortality increased with each additional organ dysfunction $(P<0.001$ for trend), and this relationship was similar for organ dysfunctions present on ward admission and those present at 24 hours after admission.

\section{Change in Organ Dysfunction over Time}

The association between in-hospital mortality and organ system dysfunction at 24 hours was dependent on the progression of the dysfunction since admission. For example, a higher proportion of patients with new or sustained dysfunction of any organ system at 24 hours died before hospital discharge when compared with patients who remained on the wards without organ dysfunction or with patients whose organ dysfunction resolved (4.6\% vs. $1.7 \%$ inhospital mortality, respectively; $P<0.001$ ). Patients with neurologic dysfunction at 24 hours had a particularly poor prognosis: new or sustained dysfunction was associated with an in-hospital mortality of $19.3 \%$ and $21.1 \%$, respectively (Figure 4 ). At 24 hours, the difference between having a new or sustained organ dysfunction was associated with similar in-hospital mortality for each organ system, with the exception of cardiovascular and pulmonary systems, where sustained organ dysfunction was associated with higher mortality than new system dysfunction $(16.3 \%$ vs. $6.7 \%$ and $23.3 \%$ vs. $13.7 \%$, respectively; $P<0.001)$.

\section{Discussion}

In this multicenter study, we found that the presence of SIRS was associated with increased mortality for ward patients. 
Ward Admission (t0)

24hrs Following Admission (t24)

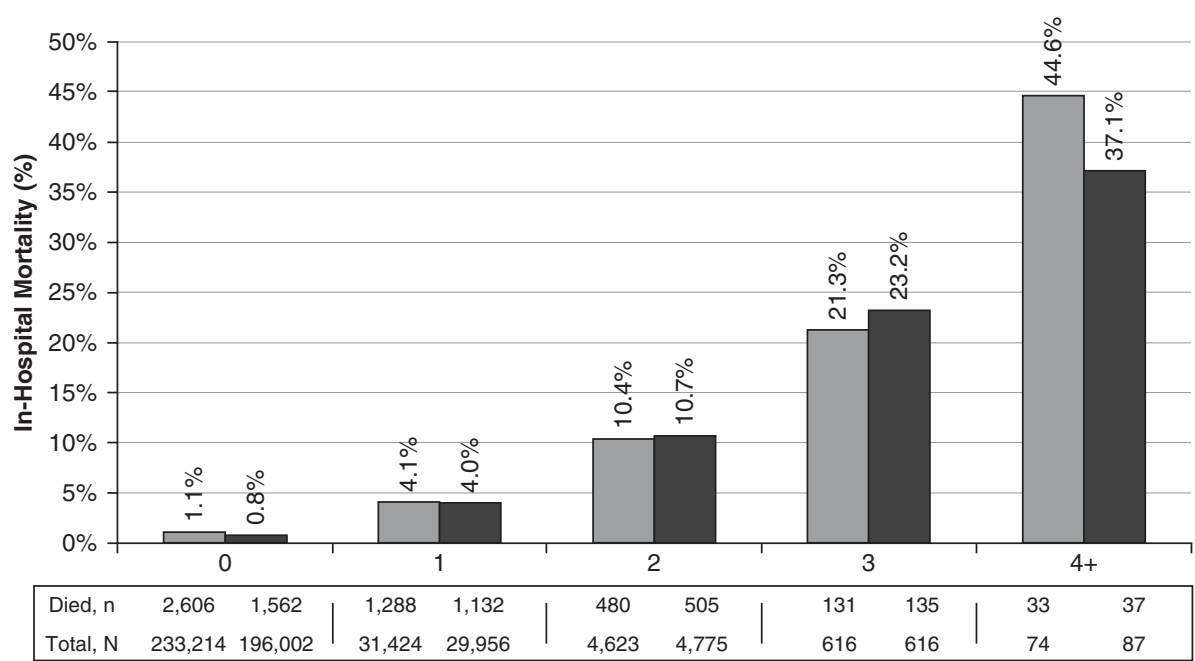

Number of Organ Dysfunctions

Figure 3. In-hospital mortality and the number of organ system dysfunctions at ward admission and 24 hours on the ward.

Importantly, we found nearly half of all patients hospitalized on the wards met SIRS criteria at least once during their stay, with the likelihood of having met SIRS criteria increasing with increasing length of stay. Our findings have important implications for inpatient sepsis screening, because a triggering rate of half of the ward population is operationally untenable and not feasible to implement.

Patients who present with sepsis on the wards have a higher mortality rate than those who present to the emergency room (26), and there is increasing interest in the early identification and treatment of these patients (17). Our study demonstrates that the SIRS criteria are too nonspecific as the initial screening mechanism for ward patients, with almost $50 \%$ meeting two of the four criteria at least once during their stay. This adds to previous literature in ICU and emergency department populations that have demonstrated similar findings (4 $27,28)$. A recent study in cardiac surgery patients also found that increasing the duration of time of continuously meeting SIRS criteria improved the prognostic ability of these criteria (27).

Although meeting these criteria for longer periods of time is a poor prognostic factor, it is unclear whether changing the duration necessary for a patient to be considered as possibly septic would benefit ward patients. Because early detection and treatment of sepsis is paramount, increasing the time before therapy in ward patients might delay necessary antibiotics, fluids, and other potentially life-saving interventions. Finally, we found that most patients who develop SIRS on the wards do so several hours to days after initial ward admission. This is likely caused by delays in obtaining laboratory results; fluctuations in

physiologic measurements over the course

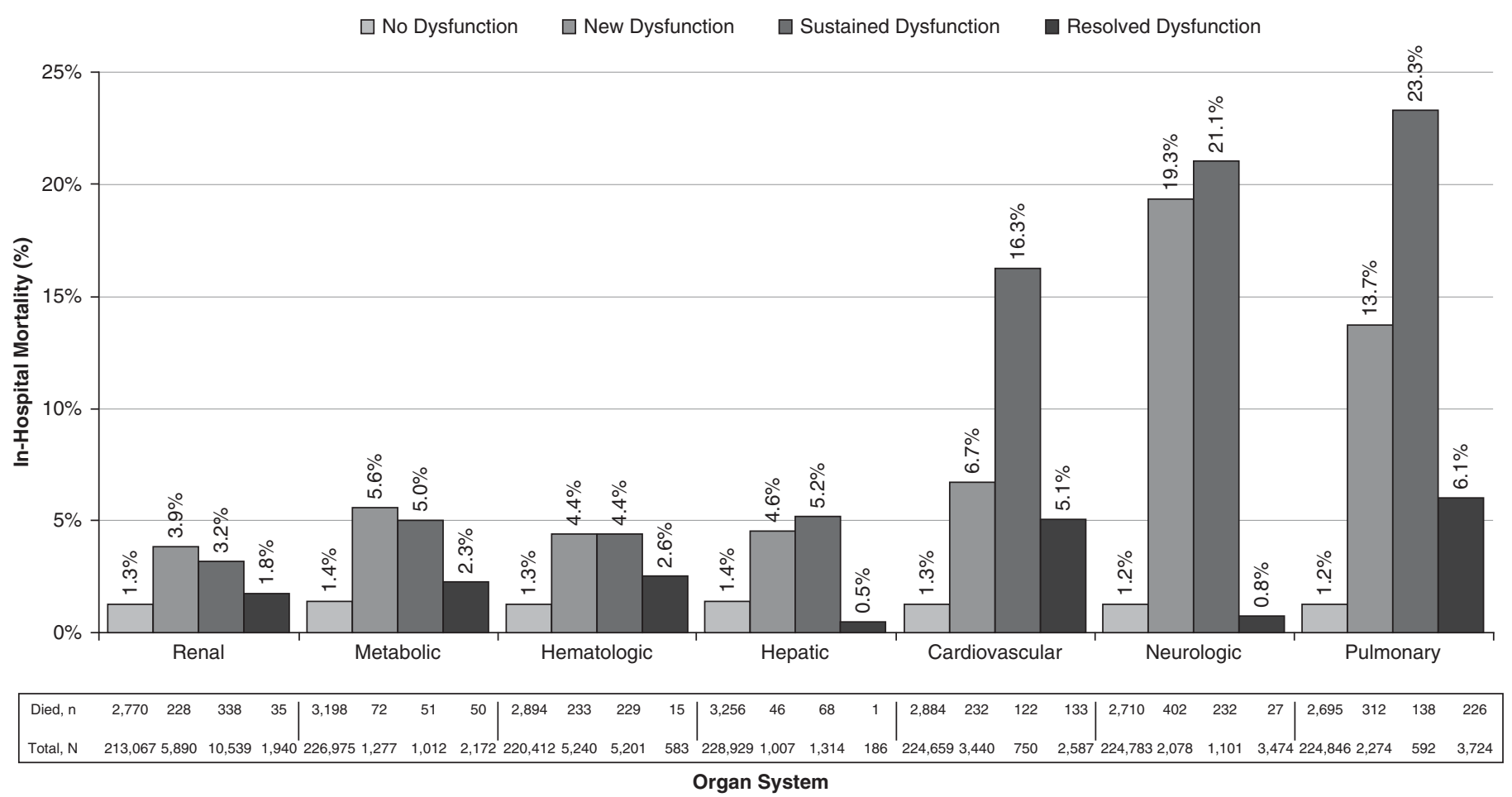

Figure 4. In-hospital mortality and the change in organ system dysfunction from ward admission to 24 hours after admission. 
of acute illness; and the fact that sicker patients, including those that suffer complications that could lead to the development of SIRS, tend to have longer lengths of stay.

Our study adds to prior literature demonstrating that in-hospital mortality increases with each additional SIRS criterion met $(4,29)$. This work extends recent findings in ICU patients that mortality increases relatively linearly with each additional criterion to patients on the general wards (29). Interestingly, we also found that different SIRS criteria had a wide range of associations with inhospital mortality, from $2 \%$ for the white blood cell criterion to $6 \%$ for the respiratory rate criterion. These findings suggest that the sensitivity and specificity of the SIRS criteria for severity of disease could be altered by changing the number of criteria that need to be met and/or altering the weight or cut-offs of the included criteria.

It is important to note that early warning scores, such as the Modified Early Warning Score and the National Early Warning Score, which are designed to identify high-risk ward patients are already in widespread use and include three of the four variables that define SIRS (30). If the goal were to first identify patients at high risk of death followed by determining which of these patients were infected, then one of these tools could be used for sepsis screening instead of SIRS criteria. In particular, the National Early Warning Score and the VitalPAC Early Warning Score have been shown to be more accurate than other vital sign-based scores, and our group and others have demonstrated that accuracy can be further improved by adding laboratory results and other variables $(21,30,31)$. Similar associations were seen with the presence of different organ dysfunctions, with pulmonary and cardiovascular dysfunction having the worst prognosis when present on ward admission. Additionally, the risk of inhospital mortality in patients with neurologic dysfunction increased dramatically from $6 \%$ when present on admission to $20 \%$ when present at 24 hours after admission. This important finding illustrates that the presence of neurologic dysfunction after 24 hours of therapy on the wards should alert caregivers that the patient has a particularly poor prognosis.

To our knowledge, our study is the first to investigate the associations between inhospital mortality and the change in organ dysfunction over time in the general ward setting. We found that patients without evidence of organ dysfunctions at both ward admission and 24 hours after admission had the best prognosis, followed by patients who presented with an organ dysfunction that subsequently resolved. As noted, patients with neurologic dysfunction on the wards 24 hours after admission had a worse prognosis than those with any other organ dysfunction. This prognosis was similar whether the patient presented with neurologic dysfunction on admission or whether this dysfunction was newly present at 24 hours. These findings not only provide important prognostic information, but also suggest that screening efforts for high-risk patients may need to use different criteria depending on how long the patient has been admitted.

Our study has several limitations. First, because this study was performed in a ward population, the criteria used in the study had to be modified because some tests, such as arterial blood gases, are not commonly performed in these patients. In addition, band percentages and $\mathrm{Pa}_{\mathrm{CO}_{2}}$ were not available so this could not be used as part of the white blood cell criterion. However, our criteria were similar to those used in studies in other settings, and not including bandemia and $\mathrm{Pa}_{\mathrm{CO}_{2}}$ resulted in an underestimation of the proportion of patients who would meet SIRS and organ dysfunction criteria. Furthermore, it is likely that the frequency of vital sign and laboratory results differ based on a patient's severity of illness. This would also alter the estimated incidence of the SIRS compared with a population where these values were always checked at the same interval regardless of severity of illness.

Second, because of the nature of this large cohort study, we were unable to determine a caregiver's suspicion for infection, so our study focused on SIRS and organ dysfunctions and not sepsis specifically. The high proportion of patients meeting SIRS criteria does have significant implications for sepsis screening in ward patients because these criteria are often used in screening tools. We also found that patients with SIRS had a higher number of hospital admissions during the study period than patients who never developed SIRS. This finding is limited by the fact that some of the patients not readmitted to the study hospitals may have been admitted to hospitals not included in our dataset and that previous admission data were unavailable before the start of the study period at each hospital. Finally, it is likely that the prognosis of SIRS and organ dysfunction criteria differs based on the patient's diagnosis (e.g., pneumonia vs. urosepsis). We did not have access to these details in this study and further research into this area is warranted.

In conclusion, we found that almost half of patients hospitalized on the wards developed SIRS at least once during their ward stay. Our findings suggest that screening ward patients using SIRS criteria for the early identification of sepsis would be time-consuming and impractical. This study also provides insight into the prognostic ability of different organ dysfunctions and their evolution over time for patients on the wards.

\section{Author disclosures are available with the text} of this article at www.atsjournals.org.

Acknowledgment: The authors thank Timothy Holper, Justin Lakeman, and Contessa Hsu for assistance with data extraction and technical support; Poome Chamnankit, MS, CNP, Kelly Bhatia, MSN, ACNP, and Audrey Seitman, MSN, ACNP for performing manual chart review of cardiac arrest patients; and Nicole Twu for administrative support.

\section{References}

1. Bone RC, Balk RA, Cerra FB, Dellinger RP, Fein AM, Knaus WA, Schein RM, Sibbald WJ; The ACCP/SCCM Consensus Conference Committee. American College of Chest Physicians/Society of Critical Care Medicine. Definitions for sepsis and organ failure and guidelines for the use of innovative therapies in sepsis. Chest 1992;101:1644-1655.
2. Rivers E, Nguyen B, Havstad S, Ressler J, Muzzin A, Knoblich B, Peterson E, Tomlanovich M; Early Goal-Directed Therapy Collaborative Group. Early goal-directed therapy in the treatment of severe sepsis and septic shock. N Engl J Med 2001;345:1368-1377.

3. Yealy DM, Kellum JA, Huang DT, Barnato AE, Weissfeld LA, Pike F, Terndrup T, Wang HE, Hou PC, LoVecchio F, et al.; ProCESS Investigators. A randomized trial of protocol-based care for early septic shock. N Engl J Med 2014;370:1683-1693. 
4. Shapiro N, Howell MD, Bates DW, Angus DC, Ngo L, Talmor D. The association of sepsis syndrome and organ dysfunction with mortality in emergency department patients with suspected infection. Ann Emerg Med 2006;48:583-590, e1.

5. Hebert PC, Drummond AJ, Singer J, Bernard GR, Russell JA. A simple multiple system organ failure scoring system predicts mortality of patients who have sepsis syndrome. Chest 1993;104:230-235.

6. Marshall JC, Cook DJ, Christou NV, Bernard GR, Sprung CL, Sibbald WJ. Multiple organ dysfunction score: a reliable descriptor of a complex clinical outcome. Crit Care Med 1995;23:1638-1652.

7. Vincent JL. Dear SIRS, I'm sorry to say that I don't like you.... Crit Care Med 1997;25:372-374.

8. Peake SL, Delaney A, Bailey M, Bellomo R, Cameron PA, Cooper DJ, Higgins AM, Holdgate A, Howe BD, Webb SA, et al.; ARISE Investigators; ANZICS Clinical Trials Group. Goal-directed resuscitation for patients with early septic shock. N Engl J Med 2014;371:1496-1506.

9. Nguyen HB, Corbett SW, Steele R, Banta J, Clark RT, Hayes SR, Edwards J, Cho TW, Wittlake WA. Implementation of a bundle of quality indicators for the early management of severe sepsis and septic shock is associated with decreased mortality. Crit Care Med 2007:35:1105-1112.

10. Russell JA, Singer J, Bernard GR, Wheeler A, Fulkerson W, Hudson L, Schein $R$, Summer W, Wright $P$, Walley KR. Changing pattern of organ dysfunction in early human sepsis is related to mortality. Crit Care Med 2000;28:3405-3411.

11. Alberti C, Brun-Buisson C, Burchardi H, Martin C, Goodman S, Artigas A, Sicignano A, Palazzo M, Moreno R, Boulmé R, et al. Epidemiology of sepsis and infection in ICU patients from an international multicentre cohort study. Intensive Care Med 2002;28:108-121.

12. Padkin A, Goldfrad C, Brady AR, Young D, Black N, Rowan K. Epidemiology of severe sepsis occurring in the first $24 \mathrm{hrs}$ in intensive care units in England, Wales, and Northern Ireland. Crit Care Med 2003;31:2332-2338.

13. Vincent JL, Sakr Y, Sprung CL, Ranieri VM, Reinhart K, Gerlach H, Moreno R, Carlet J, Le Gall JR, Payen D; Sepsis Occurrence in Acutely III Patients Investigators. Sepsis in European intensive care units: results of the SOAP study. Crit Care Med 2006;34:344-353.

14. Esteban A, Frutos-Vivar F, Ferguson ND, Peñuelas O, Lorente JA, Gordo F, Honrubia T, Algora A, Bustos A, García G, et al. Sepsis incidence and outcome: contrasting the intensive care unit with the hospital ward. Crit Care Med 2007;35:1284-1289.

15. Liu V, Kipnis P, Rizk NW, Escobar GJ. Adverse outcomes associated with delayed intensive care unit transfers in an integrated healthcare system. J Hosp Med 2012;7:224-230.

16. Escarce JJ, Kelley MA. Admission source to the medical intensive care unit predicts hospital death independent of APACHE II score. JAMA 1990;264:2389-2394.

17. Sawyer AM, Deal EN, Labelle AJ, Witt C, Thiel SW, Heard K, Reichley RM, Micek ST, Kollef MH. Implementation of a real-time computerized sepsis alert in nonintensive care unit patients. Crit Care Med 2011;39:469-473.
18. Surviving Sepsis Campaign. Evaluation for severe sepsis screening tool [accessed 2014 Nov 5]. Available from: http://www.survivingsepsis. org/SiteCollectionDocuments/ScreeningTool.pdf

19. The UK Sepsis Trust. Clinical toolkits [accessed 2014 Dec 1]. Available from: http://sepsistrust.org/info-for-professionals/clinicaltoolkits/

20. Churpek MM, Zadravecz FJ, Winslow C, Howell MD, Edelson DP. Incidence and prognostic value of the systemic inflammatory response syndrome and organ failures in ward patients [abstract] Am J Respir Crit Care Med 2015;191:A3987.

21. Churpek MM, Yuen TC, Winslow C, Robicsek AA, Meltzer DO, Gibbons $\mathrm{RD}$, Edelson DP. Multicenter development and validation of a risk stratification tool for ward patients. Am J Respir Crit Care Med 2014; 190:649-655.

22. Levy MM, Fink MP, Marshall JC, Abraham E, Angus D, Cook D, Cohen J, Opal SM, Vincent JL, Ramsay G; SCCM/ESICM/ACCP/ATS/SIS. 2001 SCCM/ESICM/ACCP/ATS/SIS International Sepsis Definitions Conference. Crit Care Med 2003;31:1250-1256.

23. Knaus WA, Wagner DP, Draper EA, Zimmerman JE, Bergner M, Bastos PG, Sirio CA, Murphy DJ, Lotring T, Damiano A, et al. The APACHE III prognostic system: risk prediction of hospital mortality for critically ill hospitalized adults. Chest 1991;100:1619-1636.

24. Shapiro NI, Howell MD, Talmor D, Donnino M, Ngo L, Bates DW. Mortality in Emergency Department Sepsis (MEDS) score predicts 1-year mortality. Crit Care Med 2007;35:192-198.

25. Royston P, Sauerbrei W. Multivariable model-building: a pragmatic approach to regression analysis based on fractional polynomials for modelling continuous variables. Chichester, England; John Wiley; 2008.

26. Lundberg JS, Perl TM, Wiblin T, Costigan MD, Dawson J, Nettleman MD, Wenzel RP. Septic shock: an analysis of outcomes for patients with onset on hospital wards versus intensive care units. Crit Care Med 1998;26:1020-1024.

27. MacCallum NS, Finney SJ, Gordon SE, Quinlan GJ, Evans TW. Modified criteria for the systemic inflammatory response syndrome improves their utility following cardiac surgery. Chest 2014;145: 1197-1203.

28. Brun-Buisson $\mathrm{C}$. The epidemiology of the systemic inflammatory response. Intensive Care Med 2000;26:S64-S74.

29. Kaukonen KM, Bailey M, Pilcher D, Cooper DJ, Bellomo R. Systemic inflammatory response syndrome criteria in defining severe sepsis. N Engl J Med 2015;372:1629-1638.

30. Smith GB, Prytherch DR, Meredith P, Schmidt PE, Featherstone PI. The ability of the National Early Warning Score (NEWS) to discriminate patients at risk of early cardiac arrest, unanticipated intensive care unit admission, and death. Resuscitation 2013;84: 465-470.

31. Prytherch DR, Smith GB, Schmidt PE, Featherstone PI. ViEWStowards a national early warning score for detecting adult inpatient deterioration. Resuscitation 2010;81: 932-937. 